\title{
Plan institucional de evaluación de aprendizajes en la Universidad Europea de Madrid
}

\author{
Eva Jiménez-García*, Sara Redondo-Duarte, María A. Ruiz-Rosillo, Juan José Rodríguez-Martín \\ Universidad Europea, C. Tajo s/n, Villaviciosa de Odón, 28670, Madrid. España. \\ (correo-e: eva.jimenez@universidadeuropea.es; sara.redondo@universidadeuropea.es; \\ mariaauxiliadora.ruiz@universidadeuropea.es; juanjose.rodriguez3@universidadeuropea.es)
}

* Autor a quien debe ser dirigida la correspondencia.

Recibido Mar. 10, 2020; Aceptado May. 8, 2020; Versión final Jul. 2, 2020, Publicado Dic. 2020

\begin{abstract}
Resumen
El presente estudio describe el plan institucional de evaluación de aprendizajes de la Universidad Europea de Madrid, describe su modelo de desarrollo y evaluación de los resultados de aprendizaje de las asignaturas. El plan pretende impulsar una cultura de evaluación sistemática de la adquisición de los resultados de aprendizaje de los estudiantes para favorecer la mejora del proceso de enseñanza-aprendizaje. Se utiliza el modelo de diseño curricular inverso y se triangulan los resultados de aprendizaje, las actividades formativas y el sistema de evaluación de 1.652 asignaturas. Basado en los resultados de la implementación del plan, se señala que la institucionalización de un plan de evaluación de aprendizajes contribuye a fomentar la cultura de la evaluación y mejora las instituciones de educación superior. Se concluye que este plan permite abordar la evaluación de los resultados de aprendizaje de los estudiantes de forma sistemática.
\end{abstract}

Palabras clave: evaluación; resultado; aprendizaje; diseño curricular inverso; competencia

\section{Institutional learning assessment plan at the Universidad Europea de Madrid}

\begin{abstract}
The present study describes the institutional learning assessment plan at the Universidad Europea de Madrid. It describes the development and assessment model for course learning outcomes. The plan aims to promote a culture of systematic acquisition assessment of student learning outcomes. This favours the continuous improvement of the teaching-learning process. A backward curriculum design model is applied to 1,652 courses. Learning outcomes, training activities, and the evaluation system are triangulated. Based on the results of the plan's implementation, it is emphasized that the institutionalization of a learning assessment plan contributes to foster assessment culture and to improve higher education institutions. It is concluded that the plan's approach presented here allows assessing student-learning outcomes systematically.
\end{abstract}

Keywords: learning assessment; outcome; backward curriculum design; competency 


\section{INTRODUCCIÓN}

Cada día es más frecuente escuchar el término assessment en el marco de la educación superior (Baird et al., 2017; Zlatkin-Troitschanskaia et al., 2015; Beaumont et al., 2011). En este ámbito, assessment es entendido como el proceso para gestionar la calidad educativa (Mateo, 2006), a través de un análisis sistemático, estructurado y reflexivo mediante el cual se recoge, organiza e interpreta la información obtenida de múltiples fuentes para determinar el nivel de efectividad de los procesos de enseñanza-aprendizaje y fundamentar la toma de decisiones responsable. Atendiendo a esta definición, hacer assessment significa realizar una valoración integral y holística del aprendizaje, y que traduciremos como valoración. Dentro de este proceso de valoración global, la evaluación es un procedimiento de análisis continuo que integra todas las actividades de enseñanza y aprendizaje dirigidas a determinar los logros alcanzados por los estudiantes. Así entendido, se considera que el proceso de valoración es imprescindible para determinar en qué medida el estudiante ha adquirido los conocimientos y las habilidades asociadas a una determinada competencia 0 conjunto de competencias.

Casanova (1998) enfatiza que toda evaluación formativa en el ámbito educativo supone la recogida de datos relativos al progreso y dificultades con las que se encuentran los alumnos en el proceso de enseñanza y aprendizaje. Por todo ello, valoración en educación superior debe poner el foco en los resultados de aprendizaje vinculados a las competencias que los estudiantes tienen que desarrollar (Havnes y Proitz, 2016; Tam, 2014); restando importancia a la impartición de unos contenidos por parte del docente, y centrándose en el logro de un determinado nivel de desempeño competencial. Solo las universidades que están a la vanguardia de la excelencia académica y de la mejora continua de la calidad de sus titulaciones han implementado un Plan de Valoración con el propósito de conseguir un desarrollo competencial integral de sus estudiantes que les aporte la máxima empleabilidad. Un ejemplo de calidad y excelencia académica son las universidades como Stanford, Berkeley o California Institute of Technology, situadas entre las diez mejores universidades a nivel mundial y que cuentan con planes de valoración.

En este contexto la Universidad Europea de Madrid (en adelante UEM) pretende impulsar en la organización una cultura de evaluación sistemática de los resultados de aprendizaje para favorecer la mejora continua del proceso de enseñanza-aprendizaje y una mayor trasparencia de los criterios de evaluación. Para ello se ha diseñado el Plan Institucional de Evaluación de Aprendizajes (en adelante PIEA). Su esencia radica en reorientar las actividades, los instrumentos y los criterios de evaluación, que tradicionalmente se han venido utilizando para evaluar a los estudiantes, de tal modo que nos permita poder establecer en qué grado han adquirido el resultado o los resultados de aprendizaje y dejar evidencia de ello (O'Brien y Brancaleone, 2011).

La valoración objetiva del logro de los resultados de aprendizaje por los estudiantes aparece como el eje que articula todo el proceso de evaluación, ya que ayuda al profesor a tomar decisiones argumentadas en relación con sus actividades educativas, a la vez que facilita la identificación de fortalezas y debilidades en los conocimientos y las habilidades de los estudiantes, y, por tanto, una mayor información acerca del nivel de dominio de una determinada competencia o conjunto de competencias (De la Mano y Moro, 2009). Por consiguiente, este Plan no pretende introducir nuevas actividades o instrumentos de evaluación, sino testarlos y consensuarlos con el resto de los profesores y evidenciar lo que se hace y el por qué se hace. Esto sitúa a los profesores como piedra angular que sustenta este Plan, siendo los garantes últimos de los resultados de aprendizajes que adquieren los estudiantes. La evaluación forma parte de la institución en su conjunto, por lo que múltiples áreas contribuyen a medir el logro en el aprendizaje curricular y extracurricular de los estudiantes. En concreto, el PIEA se adscribe al Vicerrectorado de Profesorado e Investigación y, más en concreto, a su Unidad de Innovación Educativa y Desarrollo Docente, desde la cual se despliega a toda la Universidad.

EI PIEA se basa en un proceso circular que se retroalimenta anualmente introduciendo mejoras en diferentes unidades y áreas de la Universidad, tanto docentes como no docentes. El documento del Plan es dinámico y se actualizará anualmente. Los resultados del Plan promoverán la excelencia académica y la transparencia hacia los estudiantes y agencias acreditadoras. A continuación, se describe el Plan y su modelo de desarrollo y evaluación de resultados de aprendizajes en la UEM. En primer lugar, se define el concepto de resultado de aprendizaje y los tipos de competencias; en segundo lugar, se describe el diseño curricular inverso utilizado en el PIEA y, por último, se detallan sus dos primeros niveles.

\section{DESARROLLO}

Con la creación del Espacio Europeo de la Educación Superior (en adelante EEES), iniciado en 1999 con la Declaración de Bolonia, y con el proyecto Tuning Educational Structures in Europe (González y Wagenaar, 2005), se insta a las universidades a pasar de un paradigma centrado en la enseñanza y la transmisión de conocimientos a otro centrado en el aprendizaje y en el desarrollo de competencias. Una de las principales 
características de este nuevo paradigma es que convierte al estudiante en el protagonista del sistema educativo y las competencias son el centro de los perfiles profesionales (Bolívar, 2007). En consecuencia, se pasa de un modelo de enseñanza centrado en el profesor a un modelo de enseñanza centrado en el estudiante.

\section{Competencias y resultados de aprendizaje}

La enseñanza no se reduce a la transmisión de unos conocimientos teóricos sino también al fomento de unas destrezas/habilidades y al desarrollo de unas competencias que permitan un mejor acceso al mercado de trabajo por parte de los egresados (Gijón y Crisol, 2012). Bajo esta premisa, la responsabilidad del aprendizaje recae sobre el estudiante, que asume un rol más activo, construyendo su aprendizaje de manera más autónoma, reflexiva, multidisciplinar, cooperativa y práctica. Este modelo provoca en los estudiantes un aprendizaje profundo. Por tanto, el modelo de enseñanza centrado en el estudiante significa invertir el tradicional proceso de aprendizaje centrado en el profesor y poner a los estudiantes en el centro del mismo (González y Wagenaar, 2003). Se trata de pasar de un enfoque basado en lo que el profesor enseña o en lo que el profesor debe hacer, a otro centrado en resultados de aprendizaje, es decir, en lo que los estudiantes serán capaces de comprender y hacer al terminar con éxito su proceso de aprendizaje.

A diferencia de lo que ocurre en el resto de Europa, la Agencia Nacional de Evaluación de la Calidad y Acreditación (en adelante ANECA) reconoce que el término competencias está mucho más extendido en el sistema de educación superior español que el de resultados del aprendizaje. De hecho, tanto en la legislación como en el mundo universitario, el concepto de competencias se ha utilizado casi en exclusiva para expresar lo que un estudiante debe saber, entender y ser capaz de hacer al finalizar sus estudios. En concreto, la ANECA (2015, p. 20) define una competencia como "el conjunto de conocimientos, habilidades, actitudes que se adquieren o desarrollan mediante experiencias formativas coordinadas, las cuales tienen el propósito de lograr conocimientos funcionales que den respuesta de modo eficiente a una tarea o problema de la vida cotidiana y profesional que requiera un proceso de enseñanza y aprendizaje". Además, el Marco Español de Cualificación de Educación Superior (en adelante MECES) establece cuatro tipos de competencias (tabla 1):

Tabla 1: Definición de las Competencias establecidas en MECES.

\begin{tabular}{|l|l|}
\hline Competencias & Definición \\
\hline Básicas & $\begin{array}{l}\text { Son comunes a los títulos de un determinado nivel (técnico superior, grado, máster y doctor). } \\
\text { Estas competencias se desarrollan con mayor o menor intensidad en función de las } \\
\text { características del título en cuestión. Se derivan de los saberes básicos específicos del título. }\end{array}$ \\
\hline Específicas & $\begin{array}{l}\text { Son propias de un ámbito o título y están orientadas a la consecución de un perfil específico } \\
\text { de egresado. Estas competencias deben circunscribirse a aspectos formativos y ámbitos de } \\
\text { conocimiento muy próximos al título. En general, acostumbran a tener una proyección } \\
\text { longitudinal en el título. }\end{array}$ \\
\hline Generales & $\begin{array}{l}\text { Pueden estar presentes en aquellos títulos que son habilitantes y por tanto dan acceso a } \\
\text { profesiones reguladas, y vienen determinadas por órdenes ministeriales y reales decretos. Al } \\
\text { igual que las básicas, estas competencias se desarrollan con mayor o menor intensidad en } \\
\text { función de las características del Título en cuestión y se derivan de los saberes básicos } \\
\text { específicos del Título. }\end{array}$ \\
\hline Trasversales & $\begin{array}{l}\text { Son comunes a todos los estudiantes de una misma universidad o centro universitario, } \\
\text { independientemente del Título que cursen. Las competencias transversales influyen } \\
\text { directamente en la empleabilidad y rebasan los límites de una disciplina para desarrollarse } \\
\text { potencialmente en todas ellas (transversalidad). Son necesarias para ejercer eficazmente } \\
\text { cualquier profesión y son consideradas muy importantes por los empleadores. }\end{array}$ \\
\hline
\end{tabular}

Existe una gran confusión acerca de la definición de competencia debido a las diferentes interpretaciones del término dentro de los sistemas educativos de distintos países y la utilización de los conceptos de competencia y de resultados de aprendizaje como sinónimos En el marco del EEES los resultados del aprendizaje son el producto final del proceso de enseñanza-aprendizaje. El término competencias se utiliza en un sentido más amplio permitiendo la graduación de conocimientos y habilidades o destrezas. Sin embargo, en diferentes documentos del EEES se utilizan ambos términos indistintamente, haciendo difícil su diferenciación. Algunos autores proporcionan información en cuanto a su diferenciación, en concreto algunas de las definiciones como es el caso de Harden (2007) quien entiende los resultados de aprendizaje de una asignatura como algo más sencillo y menos denso que las competencias, ya que son gestionables y se materializan en actividades de aprendizaje y evaluación. 
Por todo lo anterior, es importante conocer la definición de resultados de aprendizaje que aparece de manera explícita en el Artículo 2 del Real Decreto 1027/2011, de 15 de julio, por el que se establece el MECES como aquello que se espera que un estudiante conozca, comprenda o sea capaz de hacer tras adquirir una cualificación concreta o al finalizar su periodo de aprendizaje. Muy similar a la definición de Bingham (1999) que entiende los resultados de aprendizaje como descripciones explícitas de lo que un estudiante debe saber, comprender y ser capaz de hacer como resultado del aprendizaje. Adam (2004) concibe el resultado de aprendizaje como una declaración escrita de lo que se espera que el estudiante con éxito académico sea capaz de hacer al finalizar un módulo, asignatura o titulación.

Tal y como se aprecia, todas las definiciones anteriores coinciden en que el modelo de enseñanza se centra en el estudiante y en su aprendizaje, lo que rompe con los modelos tradicionales de educación. En el Plan Institucional de Evaluación de Aprendizajes se considera que los resultados del aprendizaje son concreciones de las competencias para un determinado nivel y que son el resultado global del proceso de enseñanzaaprendizaje. Los resultados de aprendizaje constituyen las unidades de medida que permiten evidenciar el nivel de desarrollo de dicha competencia. El desempeño del estudiante en las diferentes actividades de una asignatura permite evidenciar si ha conseguido un determinado resultado de aprendizaje. Por ello, es importante que los resultados de aprendizaje sean lo suficientemente claros, medibles y observables para que los estudiantes entiendan lo que se espera de ellos. En definitiva, tal y como indica Lawlor y Hornyak (2012) un resultado de aprendizaje debe ser simple y específico, es decir, el estudiante debe saber en todo momento qué resultados de aprendizaje debe lograr alcanzar. También el resultado debe ser medible para que los docentes y estudiantes sepan cuando se ha logrado. Esto conlleva que el resultado debe ser alcanzable y, por tanto, realista. Por último, el factor tiempo es importante para conocer si es viable alcanzar en el plazo establecido los resultados de aprendizaje previstos.

Una vez revisados los conceptos de resultados de aprendizaje y competencias, en el siguiente apartado se procede a explicar cómo se realiza el alineamiento entre los mismos. La ANECA (2013) tiene también en cuenta este proceso de alineación destacando la necesidad de identificar las competencias de cada asignatura y alinearlas con los resultados de aprendizaje esperados en la misma. Por ello, y conforme al EEES, la enseñanza en el modelo académico de la UEM se estructura en torno a la adquisición de competencias que deben reflejarse en resultados de aprendizaje evaluables en los estudiantes que cursan una determinada titulación, puesto que los resultados del aprendizaje son concreciones de las competencias para un determinado nivel y son el resultado global del proceso de enseñanza-aprendizaje (ANECA, 2013).

\section{Diseño curricular inverso}

El diseño curricular inverso consiste en comenzar la planificación de una asignatura con una especificación de los resultados del aprendizaje y utilizarlos como base para desarrollar el proceso de enseñanzaaprendizaje (Richards, 2013). Atendiendo a esta directriz, en el Plan Institucional de Evaluación de Aprendizajes, cada competencia lleva asociada un conjunto de resultados de aprendizaje, quedando los mismos definidos en términos de conocimientos (saberes de un área, tema o disciplina base del desempeño), habilidades/destrezas (capacidad para aplicar conocimientos y utilizar técnicas a fin de completar tareas y resolver problemas) y competencias (demostrada capacidad para utilizar conocimientos, destrezas y habilidades personales, sociales y metodológicas en situaciones de trabajo o estudio y en el desarrollo profesional y personal). Este proceso de alineación entre competencias y resultados de aprendizaje se lleva a cabo en la guía de aprendizaje de cada asignatura según se representa en la Tabla 2:

Tabla 2: Ejemplo de alineación entre competencias y resultados de aprendizaje.

\begin{tabular}{|l|l|}
\hline Competencias & Resultados de Aprendizaje \\
\hline \multirow{2}{*}{$\mathrm{CB} 1, \mathrm{CG} 1, \mathrm{CT} 1, \mathrm{CE} 1 \ldots$} & $\mathrm{RA} 1$ \\
\cline { 2 - 2 } & $\mathrm{RA} 2$ \\
\cline { 2 - 2 } $\mathrm{CB} 2, \mathrm{RA} 3$ \\
\cline { 2 - 2 } & RA4 4 \\
\hline
\end{tabular}

Además de realizar dicha alineación se lleva a cabo la triangulación entre resultados del aprendizaje, actividades formativas y sistemas de evaluación (ANECA, 2013), aspecto clave para garantizar la calidad de la enseñanza y para reforzar el enfoque del proceso de enseñanza y aprendizaje centrado en el estudiante. El diseño curricular inverso está alineado con estas premisas, ya que requiere que los docentes reflexionen sobre el aprendizaje específico que buscan y en las evidencias de tal aprendizaje, antes de pensar en lo que se hará en las actividades (Wiggins y McTighe, 2005).

Para llevar a cabo los pasos anteriores es necesario tener claras cuáles serán las actividades de aprendizaje que se desarrollarán en cada una de las asignaturas/módulos, entendiendo que una actividad de aprendizaje es una situación diseñada intencionalmente para promover en el estudiante un aprendizaje significativo e 
integrador y necesariamente debe contener: metodología, contenidos, recursos y escenario (Martínez et al. 2012). El siguiente paso será identificar los tipos de evidencias (figura 1) que demostrarán que el estudiante ha alcanzado o no los resultados de aprendizaje previstos.

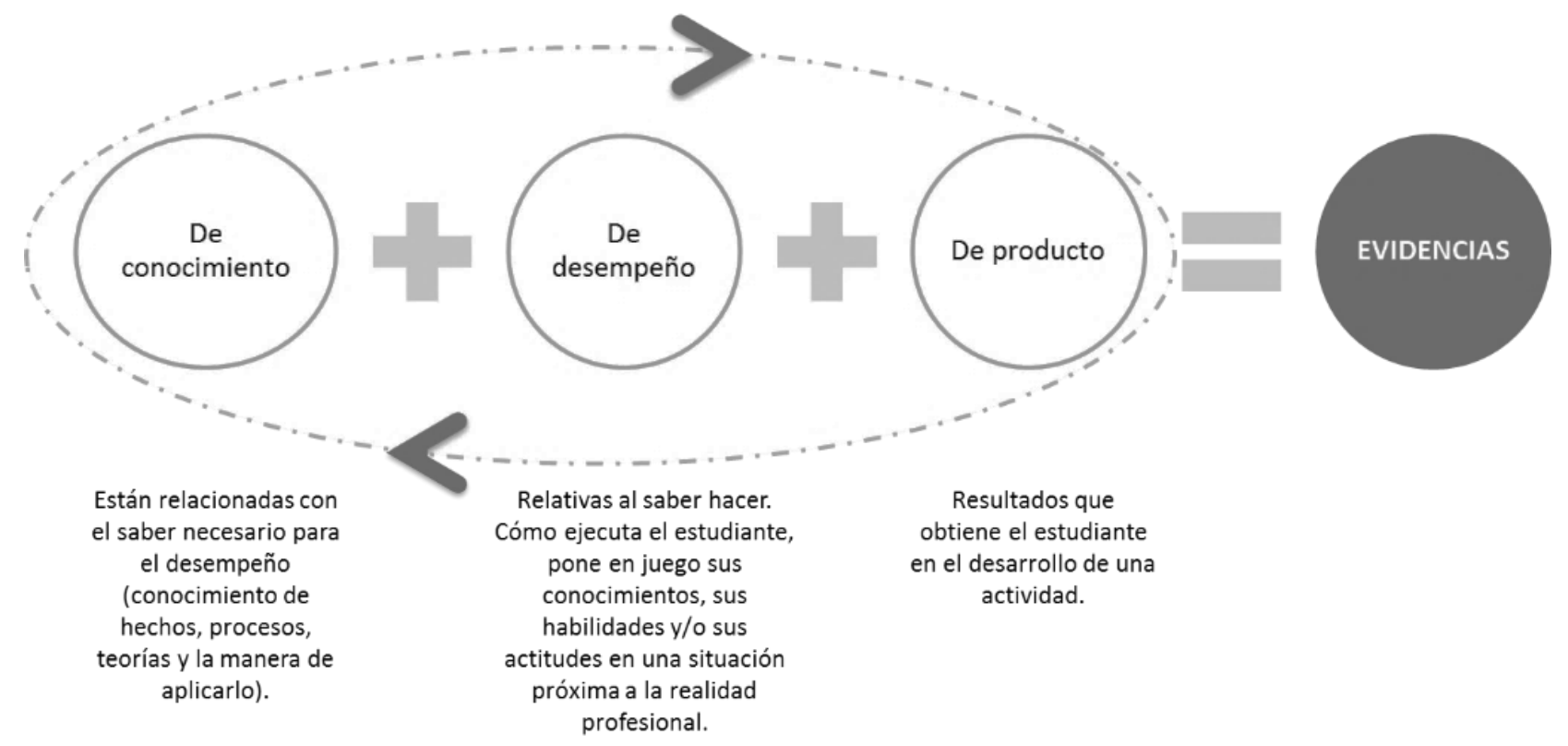

Fig. 1: Tipos de evidencias.

Este conjunto de evidencias deberá, además, ser coherente con las actividades formativas y con la evaluación que se proponga en el diseño curricular, para lo cual se requiere del alineamiento entre resultados de aprendizaje, evidencias, actividades formativas y criterios de evaluación (figura 2). Desde el punto de vista educativo, los criterios de evaluación se pueden definir como las descripciones explícitas de lo que el estudiante debe realizar para demostrar que ha conseguido el resultado de aprendizaje. Según Sadler (2005) los criterios de evaluación son un conjunto de descripciones de lo que los profesores esperan que sus estudiantes aprendan y demuestren en las evaluaciones.

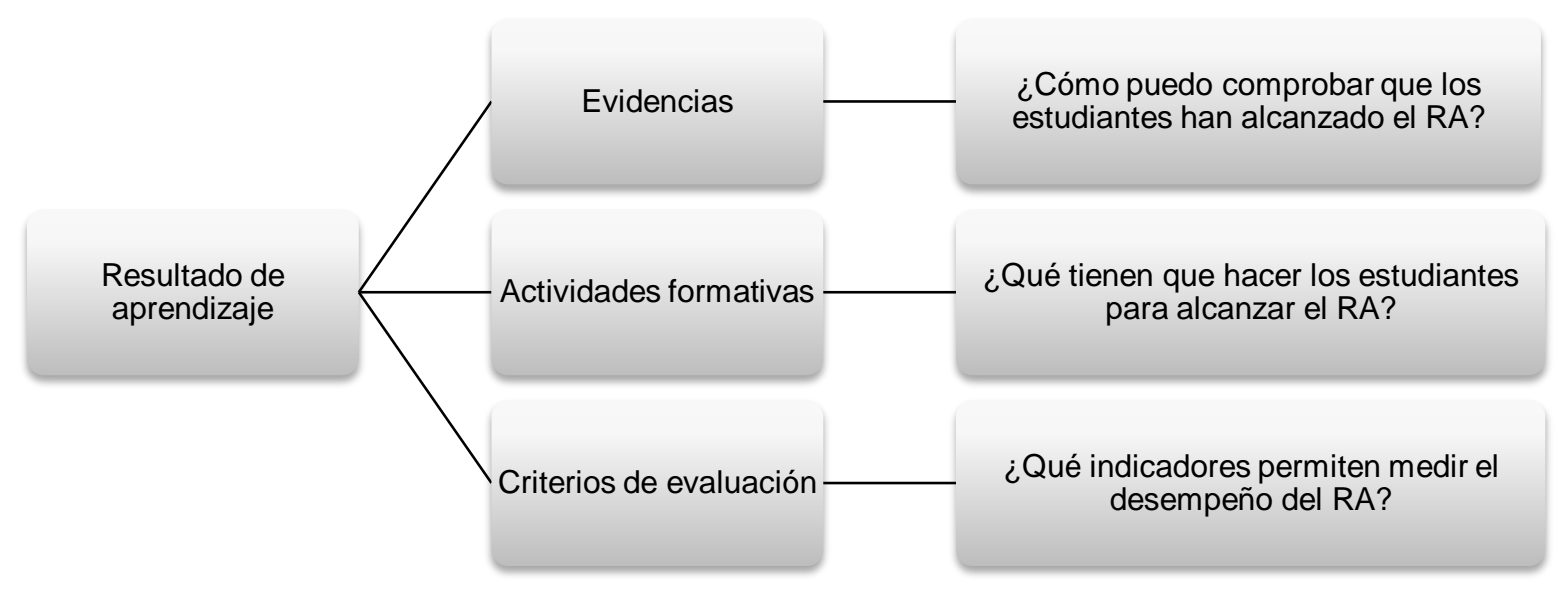

Fig. 2: Triangulación entre resultados del aprendizaje, actividades formativas, evidencias y criterios de evaluación.

De esta manera, al finalizar una asignatura, existirán evidencias que permitan identificar el nivel de logro de los conocimientos, destrezas y competencias alcanzados por el estudiante y establecer Planes de Mejora, si procede. Estas evidencias se encontrarán en los resultados de aprendizajes evaluables derivados de las actividades de la asignatura y en las propias herramientas y rúbricas utilizadas para evaluarlos. En palabras de Torres y Perera (2010, p.142), una rúbrica es "un instrumento de evaluación basado en una escala cuantitativa asociada a unos criterios preestablecidos que miden las acciones del alumnado sobre los aspectos de la tarea o actividad". En la Universidad se define la rúbrica como el instrumento de evaluación intencional del aprendizaje. A continuación, se muestra un ejemplo de plantilla para la rúbrica de evaluación de resultados de aprendizaje en una asignatura (tabla 3). 
Tabla 3: Plantilla de rúbrica de evaluación de resultados de aprendizaje.

\begin{tabular}{|l|l|l|l|l|}
\hline $\begin{array}{l}\text { Resultado de } \\
\text { Aprendizaje }\end{array}$ & $\begin{array}{l}\text { Nivel } \\
\text { insuficiente }\end{array}$ & $\begin{array}{l}\text { Nivel } \\
\text { básico }\end{array}$ & $\begin{array}{l}\text { Nivel } \\
\text { intermedio }\end{array}$ & $\begin{array}{l}\text { Nivel } \\
\text { avanzado }\end{array}$ \\
\hline RA1 & $\begin{array}{l}\text { Criterio de } \\
\text { Desempeño 1.1 }\end{array}$ & $\begin{array}{l}\text { Criterio de } \\
\text { Desempeño 1.2 }\end{array}$ & $\begin{array}{l}\text { Criterio de } \\
\text { Desempeño 1.3 }\end{array}$ & $\begin{array}{l}\text { Criterio de } \\
\text { Desempeño 1.4 }\end{array}$ \\
\hline RA2 & $\begin{array}{l}\text { Criterio de } \\
\text { Desempeño 2.1 }\end{array}$ & $\begin{array}{l}\text { Criterio de } \\
\text { Desempeño 2.2 }\end{array}$ & $\begin{array}{l}\text { Criterio de } \\
\text { Desempeño 2.3 }\end{array}$ & $\begin{array}{l}\text { Criterio de } \\
\text { Desempeño 2.4 }\end{array}$ \\
\hline RA3 & $\begin{array}{l}\text { Criterio de } \\
\text { Desempeño 3.1 }\end{array}$ & $\begin{array}{l}\text { Criterio de } \\
\text { Desempeño 3.2 }\end{array}$ & $\begin{array}{l}\text { Criterio de } \\
\text { Desempeño 3.3 }\end{array}$ & $\begin{array}{l}\text { Criterio de } \\
\text { Desempeño 3.4 }\end{array}$ \\
\hline RA4 & $\begin{array}{l}\text { Criterio de } \\
\text { Desempeño 4.1 }\end{array}$ & $\begin{array}{l}\text { Criterio de } \\
\text { Desempeño 4.2 }\end{array}$ & $\begin{array}{l}\text { Criterio de } \\
\text { Desempeño 4.3 }\end{array}$ & $\begin{array}{l}\text { Criterio de } \\
\text { Desempeño 4.4 }\end{array}$ \\
\hline
\end{tabular}

Ante estas premisas, el Plan presentado en este trabajo está basado en el diseño curricular inverso, ya que su principal principio radica en seleccionar y definir las actividades, evidencias y evaluación solo cuando han sido previamente identificados los resultados de aprendizaje que se espera que los estudiantes alcancen al finalizar la asignatura (Korotchenko et al. 2015). Igualmente, y siguiendo a Hipatia (2014, p.54, citado en Peralvo et al., 2018), el Plan considera la docencia como "el proceso organizado, intencional y sistemático que orienta los procesos de enseñanza -aprendizaje".

\section{Niveles del Plan Institucional de Evaluación de Aprendizajes}

El Plan Institucional de Evaluación de Aprendizajes se despliega a través de cinco niveles: 1) Nivel 1. Evaluación de resultados de aprendizaje en cada asignatura para garantizar que los estudiantes alcanzan el nivel de conocimientos y desarrollo competencial especificado en las respectivas Guías de aprendizaje. 2) Nivel 2. Evaluación integral y holística de los aprendizajes alcanzados en cada Título, como elemento clave en el análisis de su rendimiento. 3) Nivel 3. Evaluación de resultados de aprendizaje extracurriculares alcanzados en diferentes actividades realizadas en la Universidad, pero que no forman parte del currículum de las titulaciones. 4) Nivel 4. Evaluación de resultados de aprendizaje curriculares y extracurriculares en la Facultad/Escuela para la toma de decisiones estratégicas y la elaboración de planes de mejora. 5) Nivel 5. Evaluación de aprendizajes a nivel institucional: éste es último nivel de evaluación, donde se aprueban planes de mejora y se orienta la toma de decisiones estratégica a nivel institucional. A continuación, se describen los dos primeros niveles del Plan, pues son aquellos que se implementaron en el curso académico 2018-2019, y sobre los cuáles se dispone de resultados.

\section{Nivel 1. Evaluación de aprendizajes curriculares a nivel de asignatura}

La figura responsable de este nivel es el Coordinador de asignatura que se encarga de coordinar al resto de profesores que imparten una misma asignatura para asegurar la consecución de los resultados de aprendizaje y que éstos sean homogéneos y compartidos entre todos los estudiantes, con independencia del docente que la imparta, la modalidad o el idioma de impartición. Además, en consenso con el resto de profesores, el Coordinador de asignatura elabora la guía de aprendizaje de la asignatura. Para ello, lleva a cabo un análisis curricular enfocado en la evaluación de competencias y en el logro de los resultados de aprendizaje (Manríquez, 2012). Al término de la asignatura, el Coordinador recopila las evidencias de la evaluación de los aprendizajes en la asignatura que coordina y elabora el Plan de Mejora y coordina las acciones de mejora definidas. Para cumplir con dichas responsabilidades, el Coordinador convoca reuniones para la coordinación de su asignatura con los profesores. La reunión inicial se destina a repasar con los demás profesores el diseño curricular establecido en la asignatura (competencias, resultados de aprendizaje, contenidos, actividades, métodos docentes, dedicación del estudiante, criterios de evaluación/ rúbrica, programación, entre otros aspectos). En esta reunión se consensuan las actividades, los criterios de evaluación y las rúbricas asociadas que serán utilizadas en la asignatura en cada semestre. Durante el periodo de docencia de la asignatura, las reuniones intermedias tienen la finalidad de realizar el seguimiento con los profesores que imparten la asignatura para identificar y plantear problemas y necesidades detectados durante la impartición, y formular posibles soluciones. Por último, en la reunión final realizada al término de la asignatura, el Coordinador asegurará que se abordan todas las fases de la evaluación de los resultados de aprendizaje.

En este primer nivel del Plan, la evaluación de aprendizajes se presenta como un proceso cíclico y estructurado principalmente en tres fases consecutivas. En la primera fase de Análisis de los resultados de aprendizaje en la asignatura se examina el proceso de enseñanza y aprendizaje atendiendo a los resultados de aprendizaje de la asignatura y los criterios de evaluación. Posteriormente se recogerán las evidencias del proceso de evaluación de los mismos. La finalidad, tal y como señala Villardón (2006, p.67), es entender que 
la actividad evaluativa basada en criterios y evidencias "promueve la honestidad y el respeto por los demás, favoreciendo la colaboración y el clima de confianza". En la segunda fase de Evaluación de los resultados de aprendizaje y propuesta de aspectos de mejora se estudian tanto los resultados de aprendizaje cuyo nivel de logro alcanzado no haya sido el esperado, así como los resultados cuyo desempeño haya sido sobresaliente. Para ello se analiza la información proporcionada por las evidencias con el fin de establecer el nivel de adquisición alcanzado por los estudiantes en el resultado o resultados de aprendizaje en comparación con los estándares de rendimiento previamente establecidos por los criterios de evaluación. Una vez evaluados los resultados de aprendizaje se exponen los aspectos de mejora que posibiliten la adquisición de los resultados de aprendizaje estudiados. Por último, en la tercera fase de Elaboración del Informe de Evaluación de Resultados de Aprendizaje y Plan de Mejora de la asignatura se genera el Informe, a partir de la información resultante de las fases anteriores, cuya plantilla (tabla 4) se cumplimenta para cada resultado de aprendizaje cuyo nivel de logro no ha sido el esperado.

Tabla 4: Plantilla del Informe de Evaluación de Resultados de Aprendizaje y Plan de Mejora.

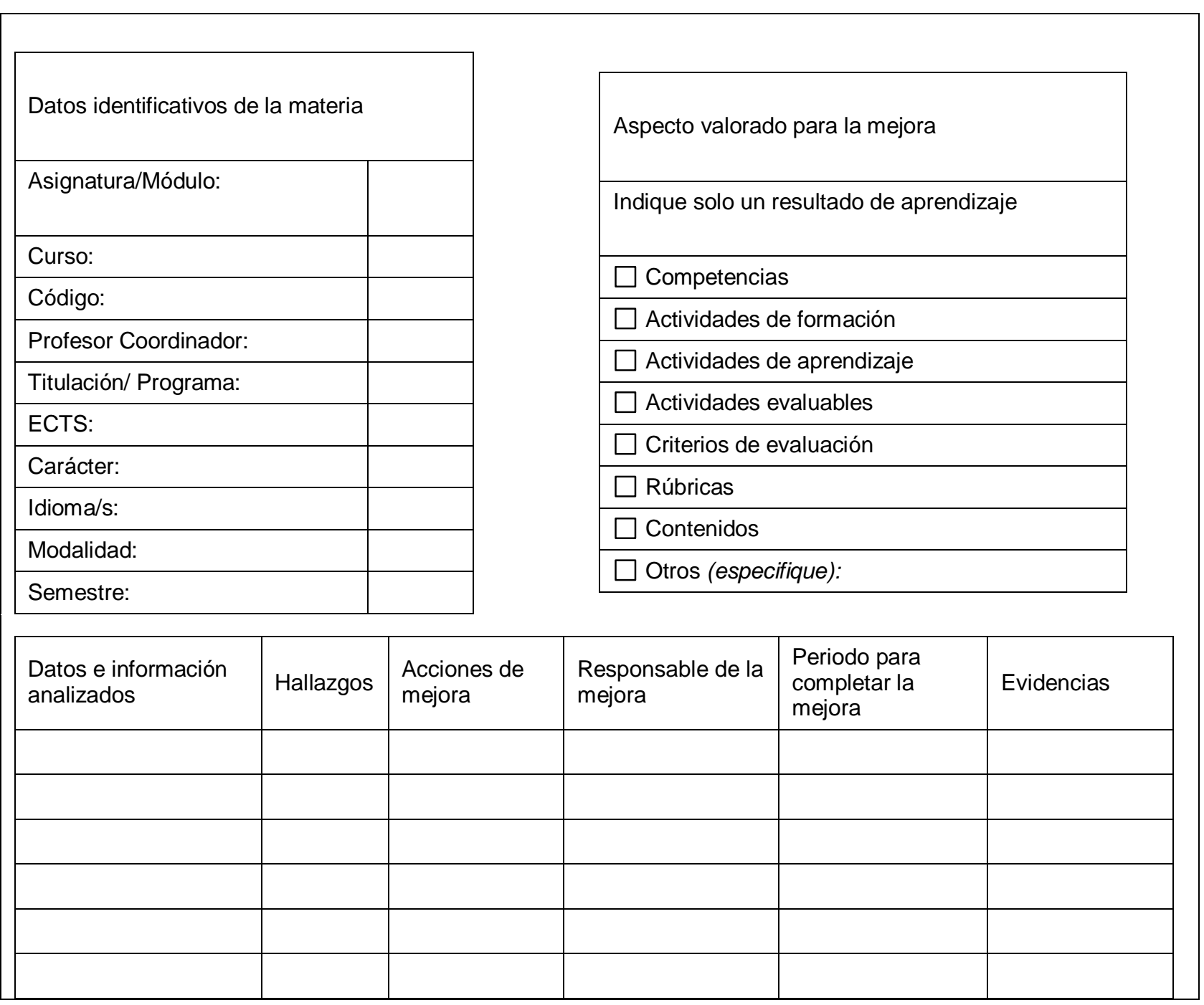

Nivel 2. Evaluación de aprendizajes en el título

La figura responsable de este nivel es el Coordinador de titulación que es el garante de mejorar continuamente la calidad del proceso de enseñanza-aprendizaje de su titulación. La evaluación a nivel de titulación, tal y como señala González (2000), requiere una perspectiva integral, holística y multifacética, que ponga de manifiesto la complejidad y riqueza del aprendizaje. Por ello, la misión principal del Coordinador de titulación es llevar a cabo el ciclo de evaluación integral y holística de los aprendizajes del título en cada curso académico y proponer acciones de mejora para el siguiente curso. Sus responsabilidades respecto al Plan son la coordinación de los equipos docentes y la supervisión de los procesos de evaluación de aprendizajes de cada asignatura para posteriormente elaborar el Informe de Evaluación de Aprendizajes y el Plan de Mejora del título. Asimismo, se encarga de revisar los avances logrados en términos de indicadores y resultados, cerrando con ello el ciclo de evaluación de aprendizajes en la titulación. 
Para cumplir con dichas responsabilidades, el Coordinador de titulación revisa los Informes de Evaluación de Resultados de Aprendizaje y Planes de Mejora elaborados por los Coordinadores de asignatura. Durante el proceso de revisión, si lo considera pertinente, convoca reuniones con el profesorado para profundizar en aquellos aspectos más críticos y/o que requieran mayor reflexión tales como el alineamiento entre las competencias y los resultados de aprendizaje, los contenidos, los métodos docentes, las actividades y/o proyectos transversales, los criterios y los sistemas de evaluación en la asignatura. En última instancia, identifica los casos de éxito y las acciones de mejora que son recogidas en la plantilla del Informe de Evaluación de Aprendizajes y Plan de Mejora del título (tabla 5).

Tabla 5: Plantilla del Informe de Evaluación de Aprendizajes y Plan de Mejora del Título.

\begin{tabular}{|l|l|}
\hline Título: & \\
\hline Curso académico: & \\
\hline № de asignaturas evaluadas: & \\
\hline № de resultados de aprendizaje evaluados: & \\
\hline № de Informes de Evaluación del Aprendizaje y Planes de mejora: & \\
\hline
\end{tabular}

Síntesis de la evaluación de los resultados de aprendizaje críticos y acciones de mejora derivados de los Informes de Evaluación de Resultados de Aprendizaje de las asignaturas/ módulo:

\begin{tabular}{|c|c|c|c|c|c|c|c|c|}
\hline Asignatura & $\begin{array}{c}\text { Competencia/ } \\
\text { Resultado de } \\
\text { aprendizaje }\end{array}$ & $\begin{array}{c}\text { Aspecto } \\
\text { valorado } \\
\text { para la } \\
\text { mejora }\end{array}$ & $\begin{array}{c}\text { Datos } \\
\text { e } \\
\text { información } \\
\text { analizados }\end{array}$ & Hallazgos & $\begin{array}{c}\text { Acciones } \\
\text { de } \\
\text { mejora }\end{array}$ & $\begin{array}{c}\text { Responsable/s } \\
\text { de la mejora }\end{array}$ & \multicolumn{2}{|c|}{$\begin{array}{c}\text { Curso } \\
\text { académico }\end{array}$} \\
\hline & & & & & & & & \\
\hline & & & & & & & \\
\hline
\end{tabular}

\section{RESULTADOS DE LA IMPLEMENTACIÓN DE LOS NIVELES 1 Y 2 DEL PLAN}

Tras la definición de los niveles 1 y 2 del Plan, se procede a la fase de implementación que concierne al Nivel 1 y a la elaboración de las Guías de aprendizaje por parte de los Coordinadores de Asignatura. Para ello fue necesario el asesoramiento al profesorado (individualizado y en claustros), así como la formación docente para la elaboración de las Guías de aprendizaje, la evaluación y mejora de los resultados de aprendizaje, y la evaluación mediante rúbricas. Adicionalmente se elaboraron las Guías del coordinador de asignatura y de Titulación con el fin de ayudar a ambas figuras a entender mejor su cometido dentro del PIEA.

Los resultados del curso académico 2018/2019, presentados en la Tabla 6, muestran que se han elaborado un total de 1652 guías, 1104 en el caso de grado y 548 guías en posgrado. En la tabla pueden verse los resultados segmentados por cada una de las facultades/escuela de la Universidad. Además 1070 docentes de la UEM participaron en los cursos de formación. En los próximos cursos se procederá al pilotaje y despliegue del PIEA para recabar información sobre la evaluación de aprendizajes realizada a nivel de asignatura/ módulo y del Título en los Comités de Evaluación de Aprendizajes del Título.

Tabla 6: Resultados de la implementación del Nivel 1

\begin{tabular}{|l|l|l|}
\hline Facultad/Escuela & Titulación & № guías elaboradas \\
\hline \multirow{2}{*}{ Ciencias Sociales y de la Comunicación } & Grado & 323 \\
\cline { 2 - 3 } & Posgrado & 184 \\
\hline \multirow{2}{*}{ Ciencias Biomédicas y de la Salud } & Grado & 206 \\
\cline { 2 - 3 } & Posgrado & 110 \\
\hline \multirow{2}{*}{ Ciencias de la Actividad Física y el Deporte } & Grado & 154 \\
\cline { 2 - 3 } & Posgrado & 73 \\
\hline \multirow{2}{*}{ Arquitectura, Ingeniería y Diseño } & Grado & 421 \\
\cline { 2 - 3 } & Posgrado & 181 \\
\hline Total & & 1652 \\
\hline
\end{tabular}




\section{CONCLUSIONES}

A la luz de la fundamentación teórica de la evaluación de aprendizajes que respalda el diseño del Plan Institucional de Evaluación de Aprendizajes y su implantación en la UEM se extraen las siguientes conclusiones principales. 1) La institucionalización de un plan de evaluación conduce a una mayor implicación y clarificación de responsabilidades de los participantes en los procesos de evaluación y mejora del aprendizaje de los estudiantes. 2) Los distintos niveles del PIEA permiten sistematizar procesos, clarificar las evidencias que demuestran sus resultados, y concretar acciones de mejora, revisables, para la consecución de planes de acción más efectivos. 3) La adopción de procesos de evaluación cíclicos, a diferencia de la consecución de un modelo lineal, permite la revisión del propio proceso y acciones acometidas en el Plan, redundando así en la retroalimentación para la mejora continua del propio Plan. 4) La medición de la calidad formativa y de la adquisición de los resultados de aprendizajes de los estudiantes es un eje clave para asegurar la implantación exitosa de un modelo de evaluación como el planteado en el Plan Institucional de Evaluación de Aprendizajes. 5) En definitiva, todo ello, conduce la consolidación de una cultura de evaluación y mejora continua institucional sistemática desde los distintos niveles de implementación del Plan.

\section{NOTACIÓN}

ANECA = Agencia Nacional de Evaluación de la Calidad y Acreditación

$\mathrm{CB}=$ Competencia Básica

$\mathrm{CE}=$ Competencia Específica

$\mathrm{CG}=$ Competencia General

$\mathrm{CT}=$ Competencia Transversal

EEES = Espacio Europeo de la Educación Superior

MECES = Español de Cualificación de Educación

PIEA = Plan Institucional de Evaluación de Aprendizajes

RA $=$ Resultado de Aprendizaje

$\mathrm{S}=$ Semestre

UEM = Universidad Europea de Madrid

\section{REFERENCIAS}

Adam, S. Using learning outcomes. Report for United Kingdom Bologna Seminar, pp 1-2, (2004).

ANECA. Guía de apoyo para la redacción, puesta en práctica y evaluación de los resultados del aprendizaje. (2013).

ANECA. Guía de apoyo para la elaboración de la Memoria de Verificación de Títulos Oficiales Universitarios (Grado y Máster). (2015).

Baird, J. A., Andrich, D., Hopfenbeck, T. N., y Stobart, G. Assessment and learning: fields apart?, https://doi.org/10.1080/0969594X.2017.1319337, Assessment in Education: Principles, Policy \& Practice, 24(3), 317-350 (2017).

Beaumont, C., O'Doherty, M., y Shannon, L. Reconceptualising assessment feedback: a key to improving student learning?, https://doi.org/10.1080/03075071003731135, Studies in Higher Education, 36(6), 671-687(2011).

Bingham, J. Guide to Developing Learning Outcomes. The Learning and Teaching Institute Sheffield Hallam University, Sheffield Halllam University, Sheffield, USA (1999).

Bolívar, A. La planificación por competencias en la reforma de Bolonia de la educación superior: un análisis crítico. ETDEducação Temática Digital, 9(esp.), 68-94 (2007).

De la Mano, M. y Moro, G. La evaluación por competencias: propuesta de un sistema de medida para el Grado en Información y Documentación. BiD: textos universitaris de biblioteconomia i documentació, 2009, vol. 23 (2), (2009).

Casanova, M. A. Evaluación: Concepto, tipología y objetivos. La evaluación educativa. Escuela básica, 1, 67-102 (1998).

Gijón, J. y Crisol, E. La internacionalización de la Educación Superior. El caso del Espacio Europeo de Educación Superior, https://doi.org/10.4995/redu.2012.6137, Revista de Docencia Universitaria. REDU. Monográfico: Buenas prácticas docentes en la enseñanza universitaria. 10 (1), 389-414 (2012).

González, J. y Wagenaar, R. (Eds.). Tuning Educational Structures in Europe. Informe Final- Fase I. Universidad de Deusto, Bilbao, España (2003).

González, J. y Wagenaar, R (Eds.). Tuning Educational Structures in Europe. Phase-II. Universidad de Deusto, Bilbao, España (2005).

González, M. Evaluación del aprendizaje en la enseñanza universitaria. Revista Pedagogía Universitaria, Vol. 5, no2, 3155 (2000). 
Harden, R. M. Outcome-Based Education: the future is today, https://doi.org/10.1080/01421590701729930, Medical Teacher, 29(7), 625-629 (2007).

Havnes, A., y Proitz, T. S. Why use learning outcomes in higher education? Exploring the grounds for academic resistance and reclaiming the value of unexpected learning. Educational Assessment, Evaluation and Accountability, 28 (3), 205-223 (2016).

Korotchenko, T. V., Matveenko, I. A., Strelnikova, A. B. y Phillips, C. Backward design method in foreign language curriculum development. Procedia-Social and Behavioral Sciences, 215, 213-217 (2015).

Lawlor, K. B. y Hornyak, M. J. SMART Goals: How the application of smart goals can contribute to achievement of student learning outcomes. Developments in Business Simulation and Experiential Learning, 39, 259-267 (2012).

Manríquez, P. L. ¿Evaluación en competencias?, http://dx.doi.org/10.4067/S0718-07052012000100022, Estudios pedagógicos (Valdivia), 38(1), 353-366 (2012).

Martínez, M. A., Cegarra N. J., y Rubio, J. A. Aprendizaje basado en competencias, una propuesta para su evaluación. Profesorado. Revista de currículum y formación del profesorado, 16, (2), 373-386 (2012).

Mateo, J. Claves para el diseño de un nuevo marco conceptual para la medición y evaluación educativas. Revista de investigación educativa, 24(1), 165-186 (2006).

O'Brien, S. y Brancaleone, D. Evaluating learning outcomes: In search of lost knowledge, https://doi.org/10.1080/03323315.2011.535972, Irish Educational Studies, 30(1), 5-21 (2011).

Peralvo, R. C., Arias, P. A. y Merino, M. M. Retos de la docencia universitaria en el siglo XXI. Revista Órbita Pedagógica, 5(1), 09-27 (2018).

Real Decreto 1027/2011, de 15 de julio, por el que se establece el Marco Español de Cualificaciones para la Educación Superior. (BOE, de 3 de agosto).

Richards, J. C. Curriculum approaches in language teaching: Forward, central, and backward design, https://doi.org/10.1177/0033688212473293, Relc Journal, 44(1), 5-33 (2013).

Sadler, R. Interpretations of criteria-based assessment and grading in higher education, https://doi.org/10.1080/0260293042000264262, Assessment \& Evaluation in Higher Education. 30(2), 175-194 (2005).

Tam, M. Outcomes-based approach to quality assessment and curriculum improvement in higher education. Quality Assurance in Education, 22(2), 158-168 (2014).

Torres J. J., y Perera, V. H. La rúbrica como instrumento pedagógico para la tutorización y evaluación de los aprendizajes en el foro online en educación superior. Pixel-Bit, 36, 141-149 (2010).

Villardón, L. Evaluación del aprendizaje para promover el desarrollo de competencias. Educatio siglo XXI, n 24, 57-76 (2006).

Wiggins, G. P. y McTighe, J. Understanding by design, http://dx.doi.org/10.14483/calj.v19n1.11490, Alexandría: ASCD, Bogotá, Colombia (2005).

Zlatkin-Troitschanskaia, O., Shavelson, R. J., y Kuhn, C. The international state of research on measurement of competency in higher education, https://doi.org/10.1080/03075079.2015.1004241, Studies in Higher Education, 40(3), 393-411 (2015). 